\title{
Surface characterization of ICF capsule by AFM-based profilometer
}

\author{
Jie Meng ${ }^{1}$, Xuesen Zhao ${ }^{2}$, Xing Tang ${ }^{1}$, Yihao Xia ${ }^{1}$, Xiaojun $\mathrm{Ma}^{1}$, and Dangzhong Gao ${ }^{1}$ \\ ${ }^{1}$ Research Center of Laser Fusion, CAEP, Mianyang 621000, PR China \\ ${ }^{2}$ P.O.Box 413, Harbin Institute of Technology, Harbin 150001, PR China \\ (Received 8 October 2016; revised 23 January 2017; accepted 5 May 2017)
}

\begin{abstract}
Outside surface fluctuations of inertial confinement fusion (ICF) capsule greatly affect the implosion performance. An atomic force microscope (AFM)-based profilometer is developed to precisely characterize the capsule surface with nanometer resolution. With the standard nine surface profiles and the complete coverage data, 1D and 2D power spectra are obtained to quantitatively qualify the capsule. Capsule center fast aligning, orbit traces automatic recording, 3D capsule orientation have been studied to improve the accuracy and efficiency of the profilometer.
\end{abstract}

Keywords: inertial confinement fusion; target design and fabrication

\section{Introduction}

Due to Rayleigh-Taylor instability during inertial confinement fusion (ICF) experiment, the imperfection of capsule surface degrades the final symmetry of the implosion and creates mix at the pusher-fuel interface, potentially quench the ignition ${ }^{[1,2]}$. Obviously, our goal is to fabricate capsules with perfect surface through optimization of the process of fabrication. However the optimization is based on precise measurement of capsules. Therefore, the ability to precisely characterize the capsule surface is extremely important.

In the 1990s, Lawrence Livermore National Laboratory (LLNL) and General Atomics (GA) developed an atomic force microscope (AFM)-based spheremapper, for mapping the outer surface profiles of ICF capsule ${ }^{[3]}$. An average fluctuation power spectrum for capsule surface was established as the National Ignition Facility (NIF) standard for acceptance of capsule surface quality ${ }^{[4]}$. Power spectrum became a significant parameter for capsule characterization.

With the progress in capsule fabrication techniques and the increasing requirements for capsule precise characterization in ICF experiments, several surface analysis methods have been developed since $1999^{[5-7]}$. Some of them are not adequate. Optical inspection and white-light interferometer cannot detect localized defects or surface roughness less

Correspondence to: D. Gao, Research Center of Laser Fusion, CAEP, No. 64 Mianshan Road, Mianyang 621000, China. Email: dgaocn@163.com than $1 / 10$ wavelength in height. It is also difficult for these methods to detect micron-sized fluctuations if they do not have abrupt edges on a curved surface. Therefore, an AFMbased profilometer is established to measure surface geometry parameters with nanometer resolution, including an overview of capsule surface mode-power spectrum, surface roughness, roundness and sphericity parameters ${ }^{[8-10]}$. The capsule, supported on a vacuum chuck, is rotated while an AFM records the circumferential surface profile. A highprecision rotary air bearing with low speed and small diameter is developed to rotate the capsule. Several key techniques, including capsule fast aligning to rotation axis, automatic recording of surface traces in one orbit, and 3D capsule orientation, have been developed to improve the functions of profilometer.

\section{Profilometer}

The AFM is an effective tool for characterizing the topography of ICF capsules, due to its nanometer resolution and tiny acting force. However, the limited scanning range restricts its application for capsule surface profile measurement. A motion system with nanometer precision is indispensable to cooperate with AFM.

The capsule profilometer setup is shown in Figure 1. The capsule is held with a vacuum chuck while rotating in front of the horizontal AFM tip. The vacuum chuck is mounted on 


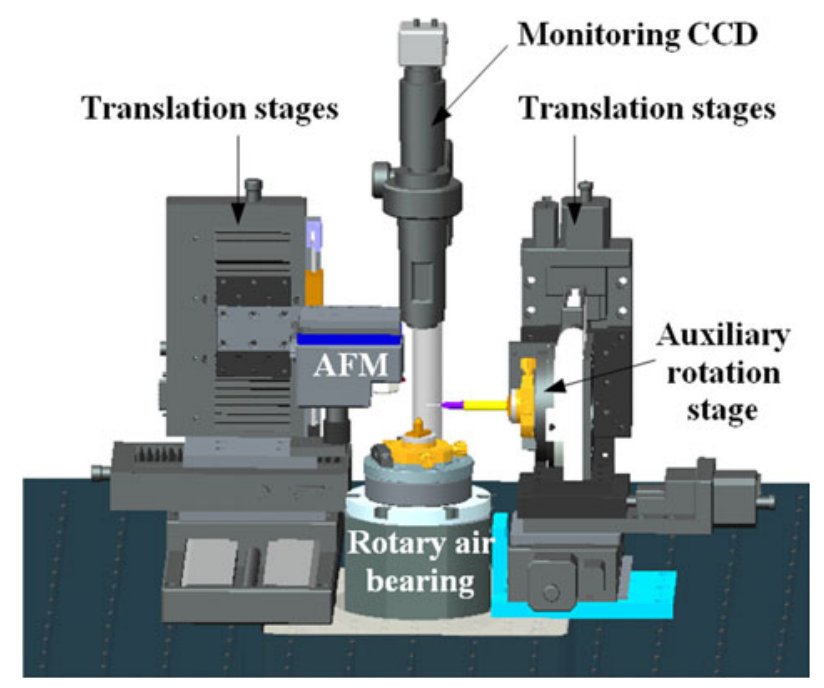

Figure 1. Schematic of capsule profilometer setup.

a vertically placed rotary air bearing which provides highprecision low-speed rotation. The shaft encoder of the rotary air bearing reports the shaft angle while the AFM records the surface height information of the capsule. Therefore the surface profile versus the circumferential angle is obtained. In order to measure arbitrary circumferential traces of a capsule, an auxiliary rotation stage is set up on the right for precise reorientation of the capsule. The horizontally laid vacuum chuck is used for mounting and removing the capsule from the rotary air bearing. Two sets of $X Y Z$ motorized translation stages precisely control the positions of the AFM tip and the auxiliary vacuum chuck, respectively. The whole apparatus is carefully vibration isolated.

The small-sized air bearing is composed of aerostatic bearing, torque motor, and circular grating for high precise rotation. The radial rotation error is less than $30 \mathrm{~nm}$. The typical rotational speed is $1-10 \mathrm{rpm}$. Uniform positioning for more than 36,000 positions of the circumference is realized, with an angular accuracy of less than $0.005^{\circ}$.

Figure 2 is a photograph of the capsule profilometer. The capsule motion and AFM tip location are monitored by CCD camera during measurement. The CCD video shots are shown in Figure 3.

\subsection{Fast alignment to rotation axis}

Before the profile measurement, the capsule center has to be aligned to the rotation axis of the air bearing. In this way, the surface fluctuations of rotated capsule will not exceed the AFM measuring range due to capsule off centeredness. The limited measuring range of AFM and the small diameter of ICF capsule make the aligning process a time-consuming and difficult task, which influences the efficiency of capsule measurement.

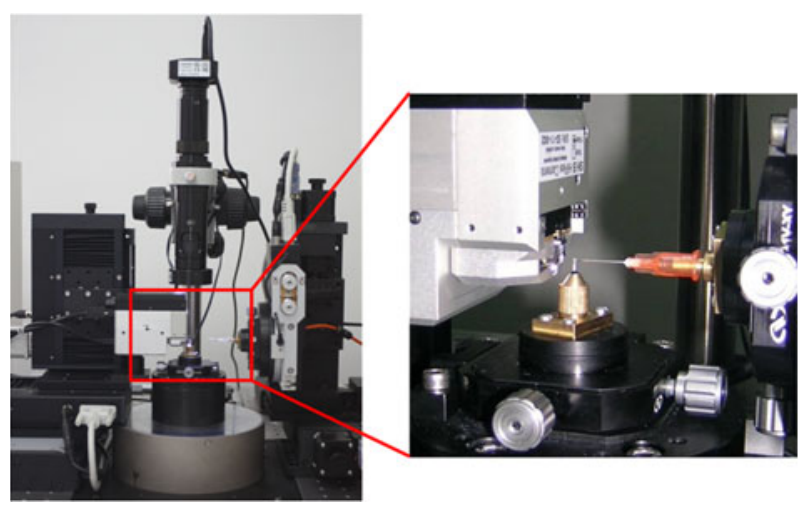

Figure 2. Photo of capsule profilometer.

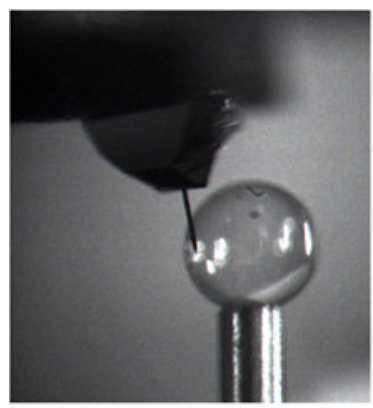

(a) Sideview

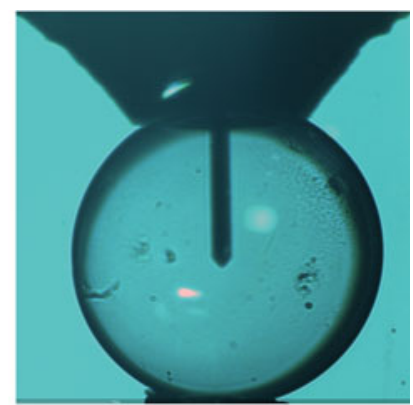

(b) Frontview
Figure 3. CCD video shots during measurement.

To solve this problem, an alignment method of capsule rotation center based on CCD image is proposed, which makes the alignment simple and fast. The aligning process is shown in Figure 4. Firstly, CCD image of the capsule is captured, the capsule edges are picked up and the first position of the capsule center is obtained [Figure 4(a)]. Secondly, the air bearing rotates $180^{\circ}$, the first step is repeated and the second position of the capsule center is obtained [Figure 4(b)]. Thirdly, the position of the rotation center is calculated according to the two positions of the capsule center and $180^{\circ}$ rotation angle. An indicative circle with the rotation center is superimposed onto the CCD video. Finally, the capsule center is aligned to the rotation center until the capsule contour is in coincidence with the indicative circle [Figure 4(c)].

After the aligning step, the eccentricity of the capsule center is restricted to less than $3 \mu \mathrm{m}$ in rotation. The aligning process takes about $3 \mathrm{~min}$, which greatly improves the profiling efficiency and guarantees a measurement progress of six capsules per day.

\subsection{Automatic recording of surface traces}

In order to improve the profiling efficiency, automatic measurement of surface traces is essential. In 3D reconstruction, we use several orbits to cover the entire capsule, and each 


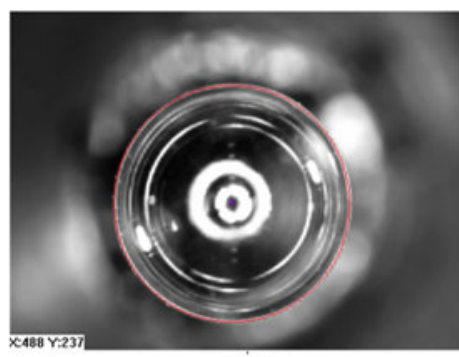

(a)

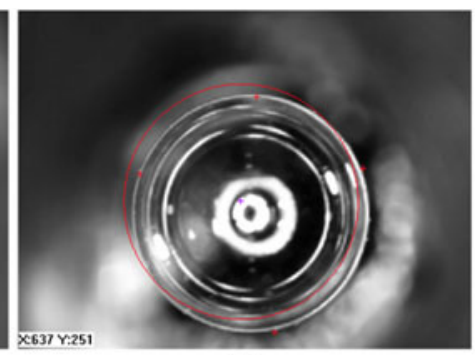

(b)

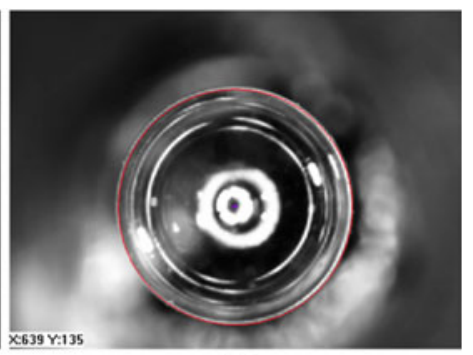

(c)

Figure 4. The process steps of capsule center alignment.

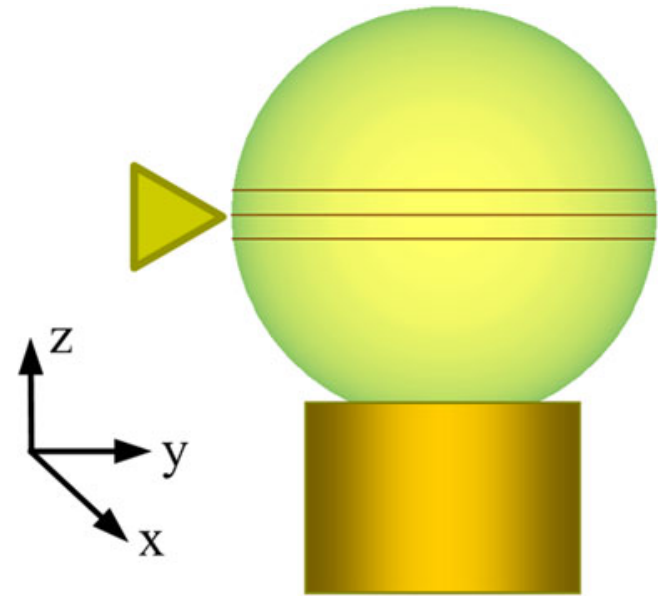

Figure 5. AFM tip and capsule.

orbit contains large amount of parallel traces. Automatic measurement of traces enlarges the covering area in single orbit, thus shortens the time for complete coverage measurement and ensures the reconstruction accuracy.

An algorithm is developed to center the AFM tip on the capsule's equator before measurement, by operating the motorized $z$-displacement and $x$-displacement stages, as shown in Figure 5. In addition, the $z$-displacement stage automatically offsets the AFM tip for parallel traces in orbit measurement and the $y$-displacement stage compensates the shift caused by $z$-offset so that the tip can be moved along the curve of the capsule.

With the developed algorithm, the surface traces in one orbit are measured automatically so long as the separation and the number of traces are set. A set of 150 measured traces with $1 \mu \mathrm{m}$ separation are shown in Figure 6. The diameter of the measured capsule is about $900 \mu \mathrm{m}$. Surface fluctuations occur in adjacent traces at the same angle, due to local defects on capsule surface.

\subsection{Precise capsule reorientation}

The standard procedure to acquire surface power spectrum of one capsule is to calculate the fluctuation power from the average of the Fourier transform of nine surface profiles ${ }^{[3]}$. The nine profiles are taken along three parallel traces in three mutually orthogonal great circles, which is the standard trace pattern [Figure 7(a)]. Another pattern is the complete coverage pattern [Figure 7(b)], in which case the capsule surface is completely covered by several polar and equatorial orbits ${ }^{[11]}$. Both patterns require precise control of capsule reorientation between two orbits measurement. Capsule reorientation is realized by two orthogonal shafting, and the auxiliary shaft is strictly perpendicular to the air bearing.

The reorientation accuracy is the key to ensure the complete surface mapping and 3D surface reconstruction. There is always a slight bobble in picking up and replacing the capsule, which influences the reconstruction accuracy. Therefore, the reorientation accuracy needs exact determination.

The suction force of the vacuum chuck is set appropriate to avoid capsule deformation and ensure the flexible operation in capsule repositioning. Based on calculations and experimental results, we designed and built a vacuum chuck with tiny suction force, which is through the shaft center. The capsule deformation caused by vacuum chuck is estimated to be one order of magnitude lower than capsule sphericity error.

The two orthogonal shafting are repeatedly adjusted according to the feature points on capsule surface, which finally guarantees the precise reorientation of the capsule. Two micropores are imposed on capsule surface by femtosecond laser ablation. As shown in Figure 8, with the obtained traces before and after reorientation operation, the reorientation accuracy can be calculated according to the position differences of micropores. As a result, the bobble in manipulating a capsule is less than $2.5^{\circ}$ in each coordinate direction.

\subsection{Noise power spectrum}

Noise power spectra are shown in Figure 9, in which the blue curve and the red curve, respectively, represent our profilometer and NIF spheremapper. The measurement uncertainty of our profilometer is calculated according to the surface roughness value of noise power spectrum, and is $1.7 \mathrm{~nm}$. 


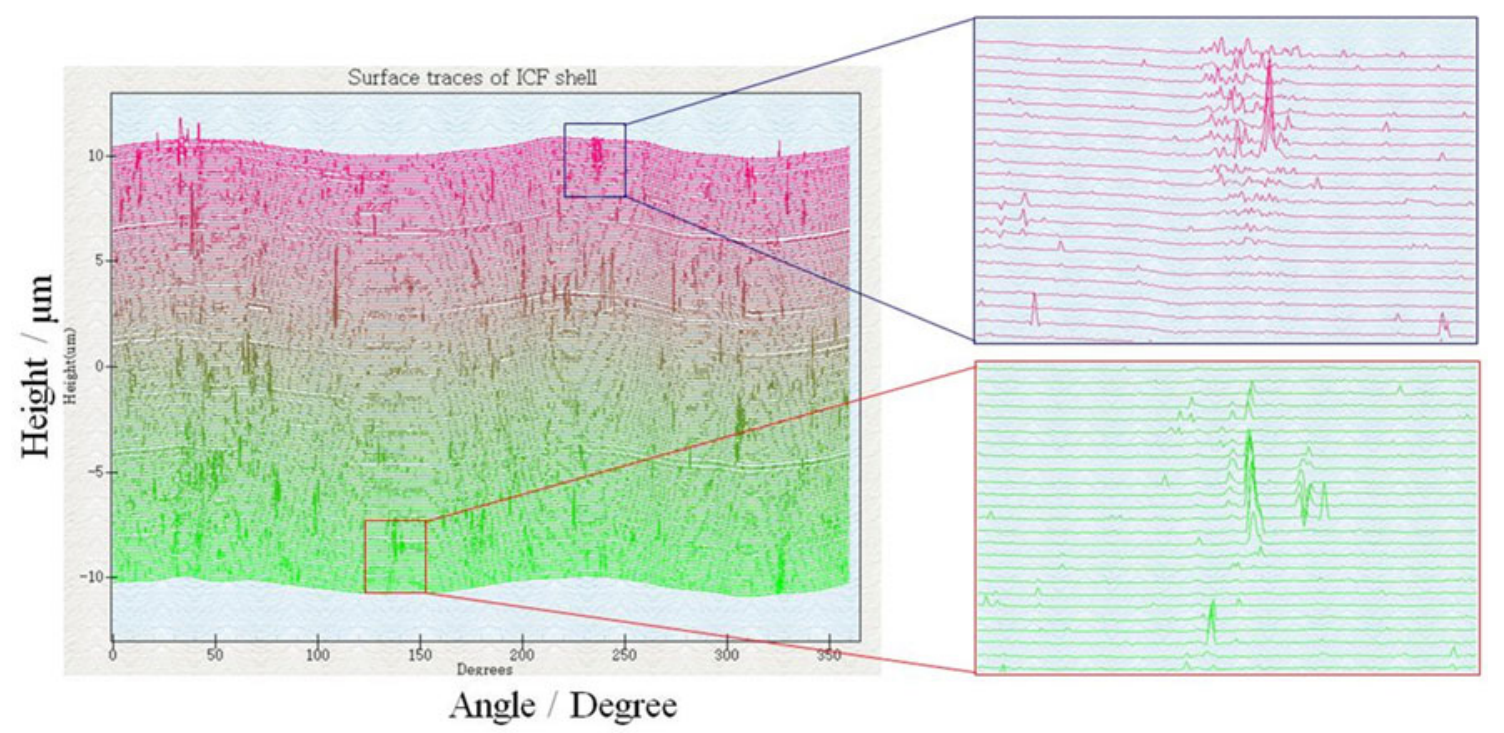

Figure 6. Measured surface traces in one orbit.

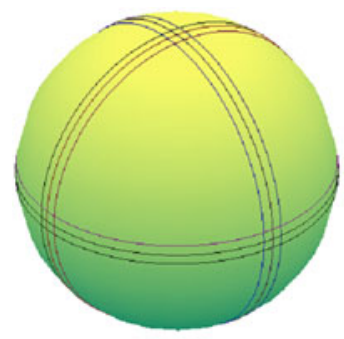

(a) standard trace pattern

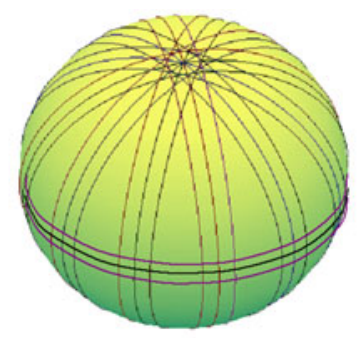

(b) complete coverage pattern
Figure 7. Trace patterns for capsule measurement.

\section{Surface topography characterization}

Based on the developed profilometer and measured surface traces of the capsule, surface geometry parameters including 1D mode-power spectrum, surface roughness, and roundness can be calculated. With the complete mapping data, the entire capsule surface can be reconstructed and 2D power spectrum is plotted. Therefore the characterization of the entire surface is realized and the capsule surface quality can be evaluated.

\subsection{Complete surface measurement and $3 D$ reconstruction}

Complete surface measurement is based on precise capsule reorientation and automatic recording of surface traces. After the recording of circumference traces in one orbit, the capsule is orientated several times with equal interval angle by the operation of two orthogonal shafting, to cover the entire surface. As shown in Figure 10, a glow discharge polymer (GDP) capsule with $2 \mathrm{~mm}$ diameter is covered by four orbits separated by $45^{\circ}$. Each orbit is $800 \mu \mathrm{m}$ wide. The small height difference between the four orbits is mainly caused by the position error at different directions. The yellow protuberant features on the sphere are due to the local defects such as spikes on the capsule surface.

The complete mapping data needs preprocessing before surface reconstruction. Profilometer only measures the deviation from a circle, not the radius itself. As a result, mode 0 (the average radius) and mode 1 (circle center) must be first retrieved for each trace to minimize the radius discrepancy and trace intersections ${ }^{[12]}$. Second, a 2D filtering algorithm based on discrete cosine transform is applied to the surface data in one orbit ${ }^{[13]}$, in order to eliminate abnormal fluctuations of adjacent traces and reduce the errors caused by environmental noise and AFM tip shifting.

After data preprocessing, all orbit data is combined and aligned according to initial measured positions, and the spherical surface map $R(\theta, \varphi)$ is obtained. The angular resolution of $R(\theta, \varphi)$ is $1^{\circ}$ with $360 \times 360$ sampling. $R(\theta, \varphi)$ is expressed as a linear combination of spherical harmonic functions ${ }^{[14]}$,

$$
R(\theta, \varphi)=\sum_{l=0}^{\infty} \sum_{m=-l}^{l} A_{l, m} \cdot Y_{l, m}(\theta, \varphi),
$$

where $A_{l, m}$ represents the spherical harmonic coefficient, and $Y_{l, m}(\theta, \varphi)$ represents the spherical harmonic function.

After the spherical harmonic expansion, the entire capsule surface is reconstructed with different orders, as shown in Figure 11. The construction results with $0-20$ orders, 21250 orders and 251-301 orders, respectively, demonstrate the surface fluctuations with low frequency, intermediate frequency and high frequency. The sphere direction is rotated (compared with Figure 10) for better demonstration. 

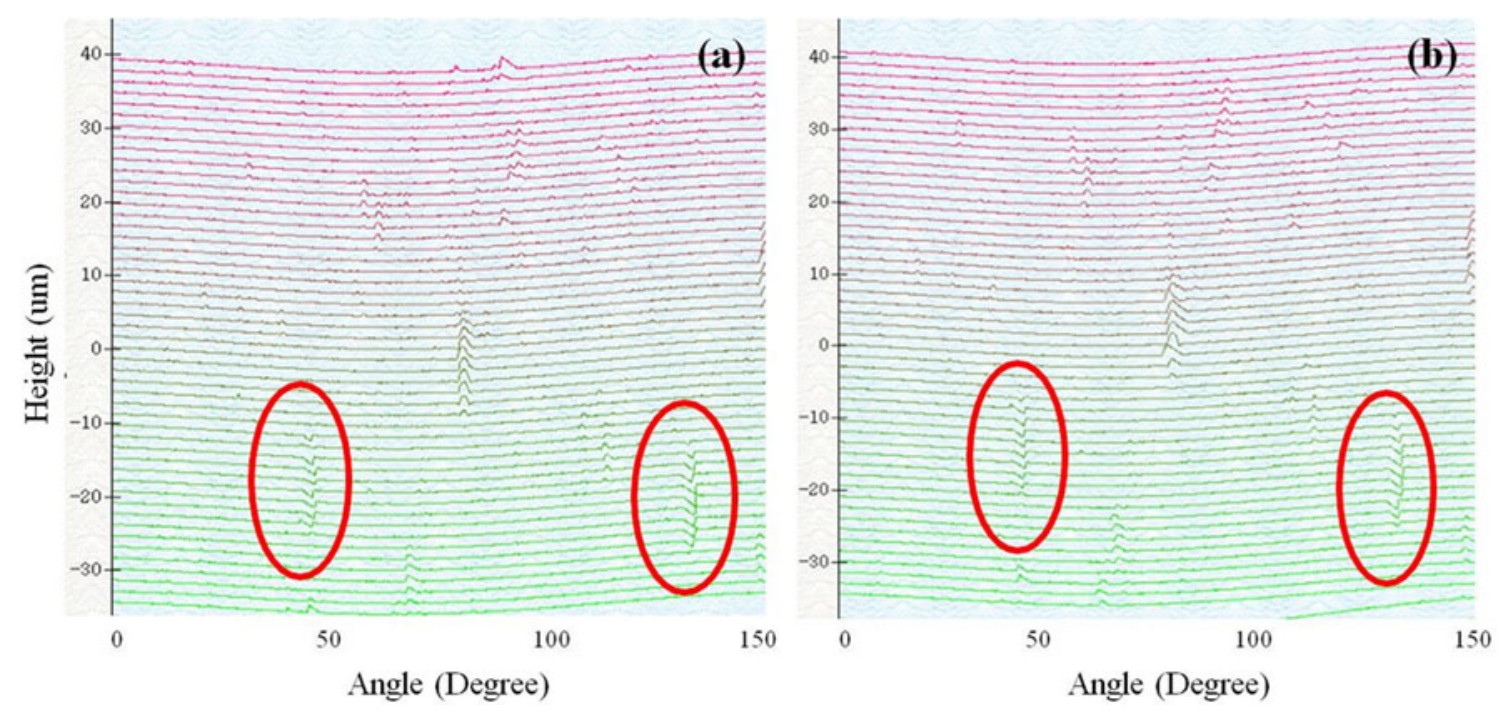

Figure 8. Traces (a) before and (b) after capsule repositioning of $360^{\circ}$.

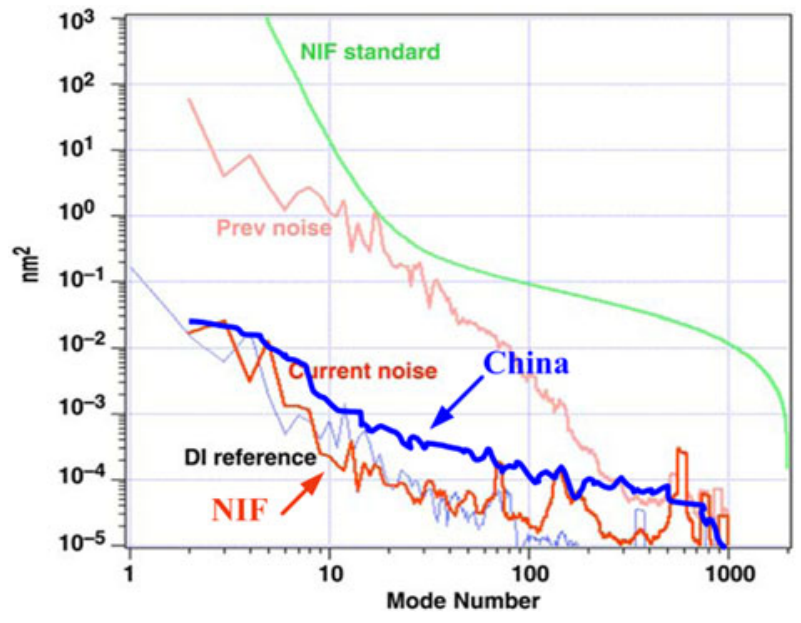

Figure 9. Noise power spectra.

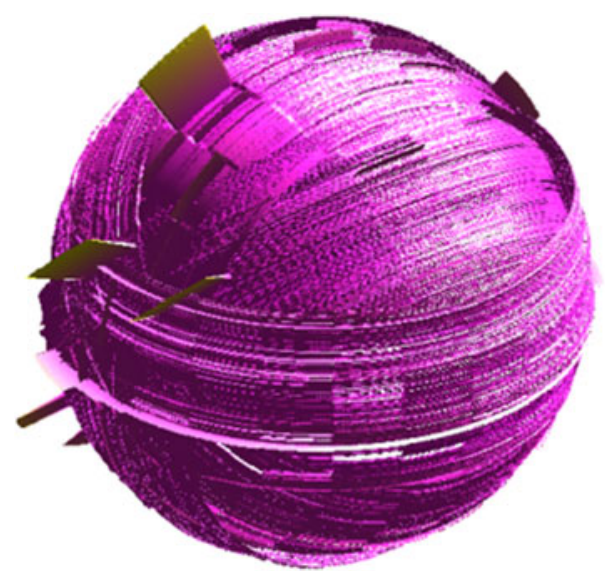

Figure 10. Orbits covering the complete surface.

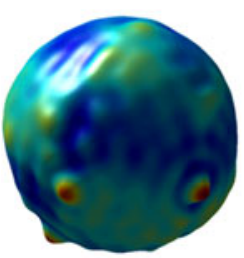

(a) $0-25$ orders

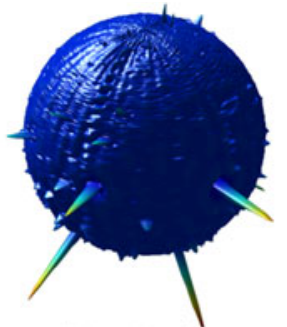

(b) 21-250 orders

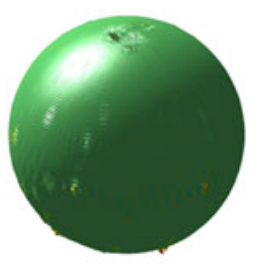

(c) 251-301 orders
Figure 11. Surface reconstruction with different orders.

\subsection{Surface characterization with mode-power spectrum}

The surface traces measured by profilometer reflect the characteristic of capsule surface. Different modes (frequencies) of capsule surface fluctuations have diverse effects on the hydrodynamic instability in implosion. To exactly evaluate the influence, the amplitude of fluctuations at each concerned mode must be acquired. Therefore, the mode-spectrum curve which gives the relationship between surface fluctuation mode and its amplitude becomes a significant parameter to estimate the capsule performance in ICF experiment.

The circumferential trace data by profile measurement is a one-dimensional discrete time sequence. The harmonic component at each mode can be obtained by Fourier transform. The 1D mode-power spectrum is plotted with the mode as horizontal coordinate and the square of the amplitude for each harmonic component as the longitudinal coordinate ${ }^{[3]}$. The 1D mode-power spectrum indicates the height fluctuations of surface harmonic components for different frequency distribution.

The measured profile traces of capsule surface and the calculated mode-power spectrum curves are shown in Figure 12. The RMS surface roughness for different mode 


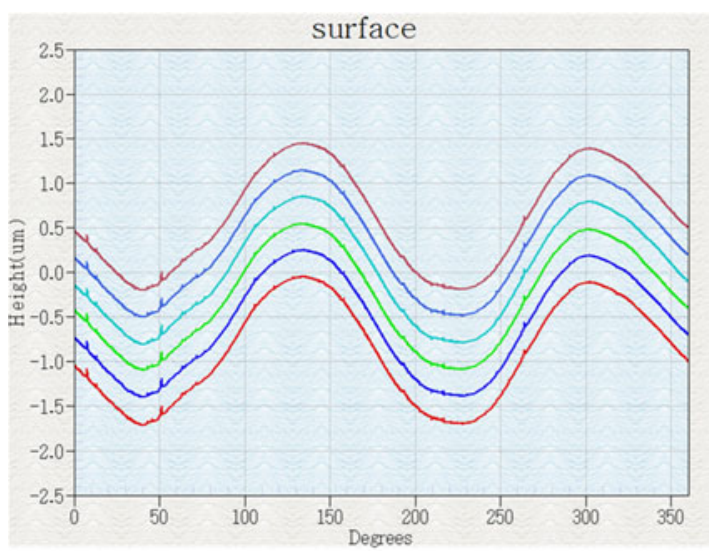

(a)

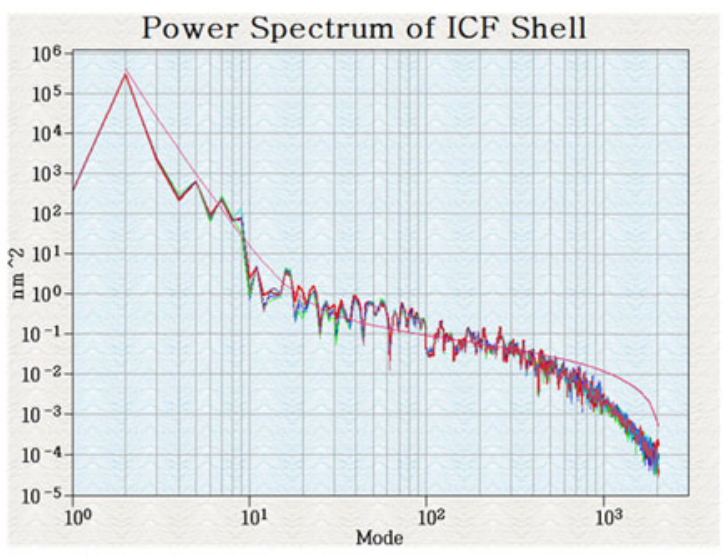

(b)

Figure 12. (a) Surface profile traces and (b) corresponding 1D mode-power spectrum.

regions can be directly calculated from the mode-power spectrum.

Compared with 1D power spectrum, 2D power spectrum is more effective for characterization of the entire capsule surface. Based on the spherical harmonic analysis of complete mapping data, the $2 \mathrm{D}$ power spectrum is calculated for entire surface evaluation.

For a $2 \mathrm{~mm}$ diameter GDP capsule, the spherical surface map $R(\theta, \varphi)$ is obtained after several orbits measurement (Figure 10). According to the theory of spherical harmonic transform, the 2D power spectrum with spherical harmonic order is calculated by,

$$
P_{2 D}(l)=\frac{1}{4 \pi} \sum_{m=-l}^{l}\left|A_{l, m}\right|^{2} .
$$

As shown in Figure 13, the 2D spherical harmonic power spectrum quantitatively reflects the frequency distribution information for capsule entire surface topography. Rapid growing of surface fluctuations for low order leads to deformation in implosion compression, and high order components will induce mixture at interface due to hydrodynamic instabilities.

\subsection{Application}

The successful establishment of capsule surface profilometer greatly promotes the fabrication of ICF capsules. On the other hand, the precise characterization of capsule surface ensures capsule quality for ICF experiments, and provides complete capsule morphology parameters for numerical simulation research. As shown in Figure 14, the amplitudes of power spectrum curves with different surface quality have difference of three orders of magnitude.

The initial morphology of the capsule is the vital factor that affecting the implosion performance. The capsule power

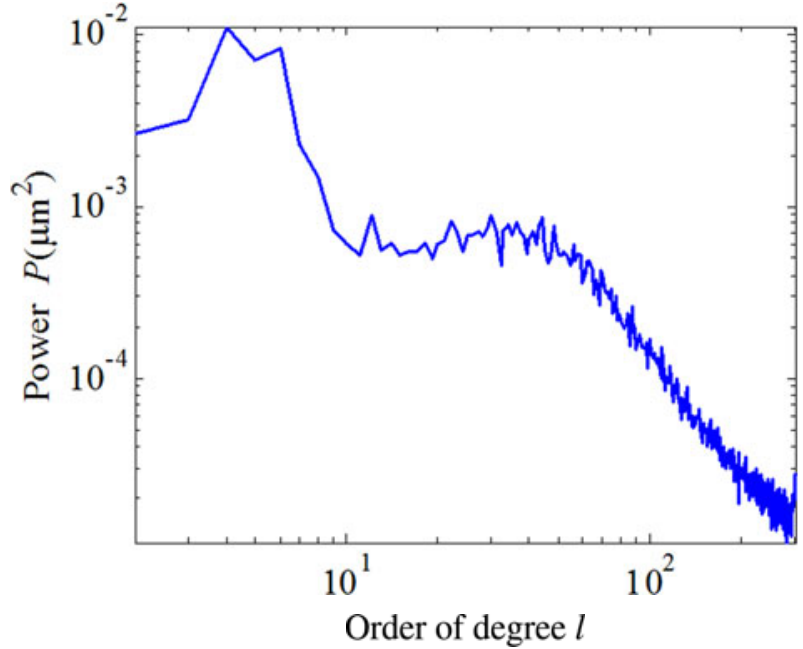

Figure 13. 2D power spectrum curve.

spectra obtained by profilometer provide significant import data for quantitative interpretation of various non-onedimensional factors on implosion performance, and comparison between the experimental results and numerical simulations.

\section{Conclusion}

Based on AFM and high-precision rotary air bearing, a capsule profilometer is developed with nanometer resolution for surface profile measurement of ICF capsule. To evaluate the capsule quality, 1D and 2D power spectra are calculated with the measured surface traces with measurement uncertainty of $1.7 \mathrm{~nm}$ and capsule reorientation error of less than $2.5^{\circ}$. With the developed techniques including capsule fast aligning to rotation axis and automatic recording of orbit traces, the measuring efficiency of six capsules per day is realized. 


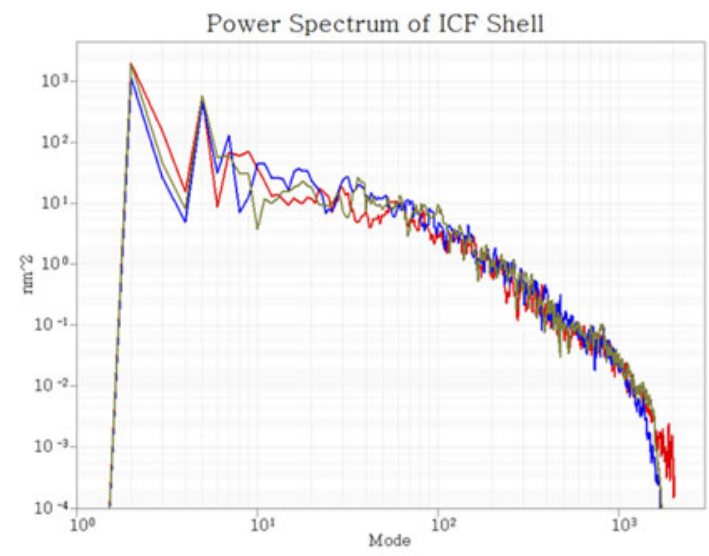

(a)

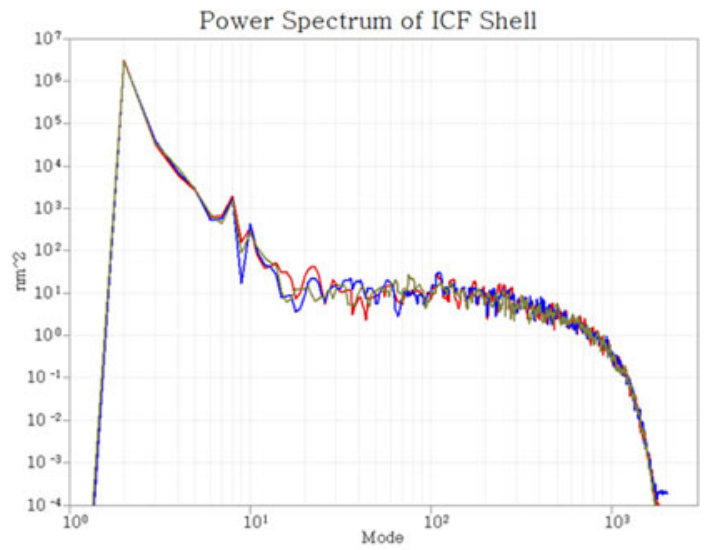

(b)

Figure 14. Power spectrum curves with (a) good surface quality and (b) bad surface quality.

\section{References}

1. R. B. Stephens, S. W. Hann, and D. C. Wilson, General Atomics Report GA-A23749 (2002).

2. S. W. Haan, P. A. Amendt, and T. R. Dittrich, Fusion Sci. Technol. 45, 69 (2004).

3. R. C. Cook, R. L. McEachern, and R. B. Stephens, Fusion Sci. Technol. 35, 224 (1999).

4. S. W. Haan, J. D. Salmonson, and D. S. Clark, Fusion Sci. Technol. 59, 1 (2011).

5. N. A. Antipa, S. H. Baxamusa, and E. S. Buice, Fusion Sci. Technol. 63, 151 (2013).

6. K. A. Moreno, S. Eddinger, and J. Fong, Fusion Sci. Technol. 55, 349 (2009).
7. B. Li, Z. Zhang, and Z. He, High Power Laser Part. Beams 27, 032024 (2015).

8. X.-S. Zhao, T. Sun, and Y.-D. Yan, Key Eng. Mater. 315-316, 796 (2006).

9. X.-S. Zhao, T. Sun, and X.-J. Ma, At. Energy Sci. Technol. 42, 833 (2008).

10. X.-S. Zhao, D.-Z. Gao, and X.-J. Ma, At. Energy Sci. Technol. 46, 1014 (2012).

11. R. B. Stephens, D. Olson, and H. Huang, Fusion Sci. Technol. 45, 210 (2004).

12. H. Huang, R. B. Stephens, and J. B. Gibson, Fusion Sci. Technol. 49, 642 (2006).

13. D. Garcia, Comput. Stat. Data Anal. 54, 1167 (2010).

14. H. Groemer, Geometric Applications of Fourier Series and Spherical Harmonics (Cambridge University Press, 1996). 